\title{
Supporting Information: Hydroxyl Radical-Coupled Electron-Transfer Mechanism of Flavin-Dependent Hydroxylases
}

Sara E. Tweedy ${ }^{+}$, Attabey Rodríguez Benítez ${ }^{+}$, Alison R. H. Narayan ${ }^{+\Phi} \perp$, Paul M. Zimmerman ${ }^{\S}$,

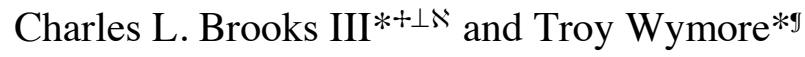

+Program in Chemical Biology, University of Michigan, Ann Arbor, MI 48109

'Department of Chemistry, University of Michigan, Ann Arbor, MI 48109

${ }^{\perp}$ Life Sciences Institute, University of Michigan, Ann Arbor, MI 48109

«Biophysics Program, University of Michigan, Ann Arbor, MI 48109

*To whom correspondence may be addressed: Email: brookscl@umich.edu or wymoret@umich.edu ; Phone: (734) 615-0401 (T.W.) or (734) 647-6682 (C.L.B.) 


\section{Table of Contents}

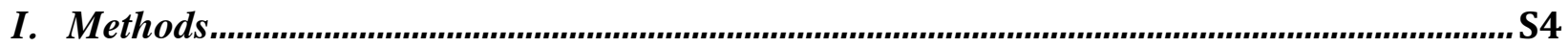

Docking of 3-methyl-orcinaldehyde to TropB ............................................................................. S4

Construction of solvated ternary complex for MD simulation ...........................................................S5

Density functional tight binding $3^{\text {rd }}$ order (DFTB3)/MM simulations.............................................. S5

Unrestricted DFT/MM potential energy scans in singlet and triplet states....................................... S6

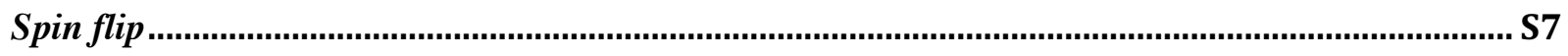

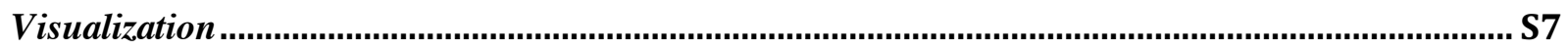

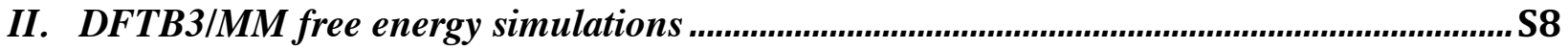

Figure S1: DFTB3/MM singlet free energy profile for hydroxylation of 3-methyl-orcinaldehyde from umbrella sampling and WHAM analysis............................................................................ S9

Figure S2: Representative structures from the A) Michaelis and B) product regions of the DFTB3/MM free energy profile....................................................................................................S10

Table S1: Distances in Michaelis complex and product structures ...............................................S11

Figure S3: Scheme illustrating hydrogen peroxide formation during DFTB3/MM US simulations

III. Multiple sequence alignment of 280 fungal flavin-dependent hydroxylases.................... S12

IV. B3LYP/MM potential energy scans checking for hysteresis ........................................... S12

Figure S4: B3LYP/MM potential energy profile for hydroxylation of 3-methyl-orcinaldehydeS13

V. Comparison of DFT/MM methods .................................................................................. S14

Table S2: Values for reaction barriers, singlet-triplet gaps, and energy changes are shown for the different QM/MM models used.................................................................................................

VI. Restricted-Active-Space n-Spin Flip results ...................................................................... S15

Molecular orbital analysis of 3-methyl-orcinaldehyde hydroxylation ............................................S15

Figure S5: Highest-occupied and lowest-unoccupied molecular orbitals from the RAS-SF

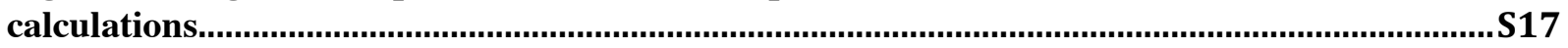

Table S3. Orbital occupancies near the transition state region according to RAS-SF .................S18

VII. Detailed analysis of mechanism from B3LYP/MM method .......................................... S19

Table S4: NBO spin density distribution in the transition state region of the B3LYP triplet

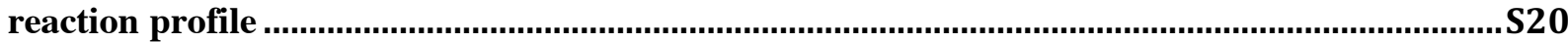

Table S5: NBO charge distribution along the B3LYP reaction profile. .......................................S21

Table S6: Distances along the B3LYP reaction profile.................................................................S22 
VIII. B3LYP/MM calculations with only the flavin and substrate represented in the QM

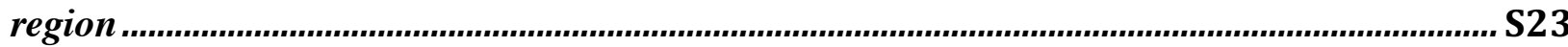

Figure S6: B3LYP/MM potential energy profile for hydroxylation of 3-methyl-orcinaldehyde with a minimal $\mathrm{QM}$ region

IX. B3LYP/MM potential energy scans of the hydroxylation reaction with C8-modified flavins.

Figure S7: B3LYP/MM potential energy profile for hydroxylation of 3-methyl-orcinaldehyde with C8-H and C8-Cl modified flavins

Figure S8: B3LYP/MM potential energy profile for hydroxylation of 3-methyl-orcinaldehyde

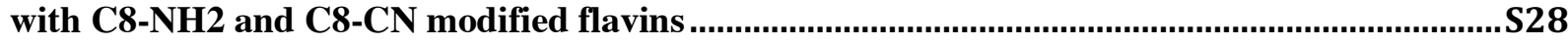

Figure S9: B3L YP-minimized structure for the C8-dimethylamino modified flavin...................S29

Table S7: Summary values for modeled modified flavins...............................................................529

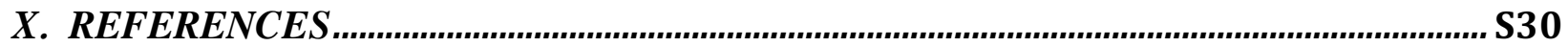


Note: The Methods are duplicated, in part, from the main text in appropriate sections for convenience.

\section{Methods}

\section{Docking of 3-methyl-orcinaldehyde to TropB}

The protocol for ligand docking of 3-methyl-orcinaldehyde [3MO] to the holo-form of TropB with the flavin cofactor in the oxidized form used the CHARMM software package ${ }^{1}$ and has been detailed in the report on the TropB structure ${ }^{2}$. The protocol is also described here since the results form, in part, a key component of modeling the TropB hydroxylation reaction.

The TropB-cofactor coordinates were obtained from the crystal structure and the protons were added with CHARMM-GUI ${ }^{3}$ according to their canonical protonation states. The tautomeric state of histidine residues was assigned in order to maximize hydrogen bonds between protein residues. The hydroperoxyflavin coordinates were simply modified from the reduced flavin by adding the hydroperoxy group to $\mathrm{C} 4 \mathrm{a}$ on the $r e$ face of the flavin. The phenolate form of $3 \mathrm{MO}$ was used for docking. Soft grid potentials ${ }^{4}$ were first calculated for all fixed elements of the receptor with probe radii corresponding to the latest CGENFF atom types ${ }^{5}$. Flexible side chains were chosen to encompass the broad active site surrounding the flavin, and included Ala55, Thr57, Leu96, Trp98, Phe119, Arg206, Leu226, Met228, Iso237, Tyr239, Asn248, Phe252, and Trp426. A random rotation of the $\mathrm{C} 1-\mathrm{C}$ (aldehyde) bond (the only rotatable bond in the substrate) was performed with the "obrotamer" algorithm in OpenBabel. The structure of 3MO was then geometry optimized using force field parameters obtained from CGENFF with minor adjustments to the charge distribution in order to delocalize the negative charge over the entire molecule and that are consistent with the charges derived from a Mulliken partitioning. The parameters are provided in a previous publication ${ }^{2}$.

The center of the ligand docking cube (the search space) was set to the average coordinates of the flexible residues, which were chosen to surround the flavin and active site. The cube dimensions were defined by the maximum coordinates of the flexible residues in each direction plus $10 \AA$. The resulting grid was a $72 \AA$ cube with a spacing of $0.5 \AA$. The $3 \mathrm{MO}$ model was then translated within the search space and randomly rotated and/or further translated. The structure was then geometry optimized with very soft and soft potentials and then removing any complexes with highly unfavorable poses ( $>200 \mathrm{kcal} / \mathrm{mol})$. At this stage, the substrate, flavin, and flexible 
side chains are represented by the CHARMM force field ${ }^{6}$ while the rest of the model is represented by the pre-computed grid potentials. A simulated annealing simulation was then performed with very soft and soft potentials (very soft: 4.5 ps of MD with increasing temperature $300-->700 \mathrm{~K}$ and 21 ps of MD decreasing temperatures $700-->300 \mathrm{~K}$; soft: 10.5 ps of MD with decreasing temperature $500-->300 \mathrm{~K}$ and finally 4.5 ps of MD with decreasing temperature $400-->50 \mathrm{~K}$ ).

Next, the entire model is represented by the CHARMM force field with a distant dependent dielectric function $(\varepsilon=3)$ while geometry optimization is performed with 50 and 100 steps using the Steepest Descent (SD) and Adopted Basis Newton Raphson (ABNR) algorithms, respectively to predict the docked pose. Final energies were calculated using generalized Born molecular volume (GBMV) implicit solvent model ${ }^{7}$. Docking poses were ranked by total energy of the complex, and low energy structures consistent with known stereochemical outcome and with a C3-

$3 \mathrm{MO}$ to $\mathrm{O} \delta$-hydroperoxyflavin distance $<4 \AA$ were considered "reactive" geometries and were considered for further analysis and simulation.

\section{Construction of solvated ternary complex for MD simulation}

A few energetically favorable docking poses with "reactive" geometries were subsequently used to construct solvated models. The coordinates of the protein, substrate, and flavin were taken directly from the docking results. In addition, oxygen atoms identified in the crystal structure as water molecules were retained in the model with protons being added with the HBUILD program in CHARMM. Bulk solvent surrounded the entire ternary complex in a cube with the size being the largest dimension of the protein plus $20 \AA$. Counterions $\left(81 \mathrm{~K}^{+}\right.$and $78 \mathrm{Cl}^{-}$ions $)$were added to the model to correspond with a 0.15 mol concentration and an overall electrically neutral model. The active-site chloride ion identified in the crystal structure was also represented though this ion immediately translates out of the active site during the simulation. The final model contained 90761 atoms.

\section{Density functional tight binding $3^{\text {rd }}$ order (DFTB3)/MM simulations}

These simulations were initiated from a snapshot of the classical MD simulation that showed the substrate poised to react with the hydroperoxyl group. This structure was geometry optimized with the CHARMM force field until the average gradient was less than $0.0001 \mathrm{kcal} \mathrm{mol}^{-}$ 
${ }^{1} \AA^{-1}$ using the adopted basis Newton-Raphson algorithm. The model was subsequently partitioned into a region represented by the DFTB3 method ${ }^{8}$ and the surroundings by the CHARMM MM force field. ${ }^{6}$ The DFTB3 region included the flavin isoalloxazine moiety (plus two carbon centers beyond this towards the adenine moiety), the 3MO substrate, the side chains of Arg206, Tyr239, and Asn248 along with 7 proximal water molecules. Three hydrogen link atoms placed along the $\mathrm{C} \beta-\mathrm{C} \alpha$ axis at a distance of $1.09 \AA$ from the $\mathrm{C} \beta$ atom for the protein side chains. The fourth link atom is placed along the second $\mathrm{C}-\mathrm{C}$ bond from $\mathrm{N} 10$ in the flavin cofactor. A total of 125 atoms were represented by the DFTB3 method. The MM geometry optimized structure was further geometry optimized with the DFTB3/MM hybrid potential until the average gradient was less than $0.0001 \mathrm{kcal} \mathrm{mol}^{-1} \AA^{-1}$ using the adopted basis Newton-Raphson algorithm. A potential energy scan for the hydroxylation reaction was performed using the mass-weighted distance difference between the peroxy $\mathrm{O} \delta-\mathrm{Op}$ bond and the forming $\mathrm{O} \delta$-C 3 bond [Reaction coordinate $=0.571(\mathrm{O} \delta$ Op-distance $)-0.429$ (C3-O $\delta$-distance) $]$ as the reaction coordinate. A restraint of $400 \mathrm{kcal} \mathrm{mol}^{-1}$ $\AA^{-2}$ was placed on the reaction coordinate in order to drive the model from the reactant to product state. Atoms further than $32 \AA$ from the substrate (defined in the initial Michaelis complex pose) were fixed. Thirty-three umbrella sampling windows were used with spring constants ranging from 50-250 kcal mol ${ }^{-1} \AA^{-2}$ to enhance sampling (150 x 16 windows, $200 \times 7$ windows, $250 \times 5$ windows, $100 \times 1$ window, $125 \times 2$ windows, 50 x 1 window, $175 \times 1$ window). Each window was simulated for $50 \mathrm{ps}$ with a $1 \mathrm{fs}$ timestep using a Langevin thermostat (fbeta = 5) at $298 \mathrm{~K}$. Analysis was performed on the last 40 ps of each simulation using the weighted histogram analysis method $\left(\mathrm{WHAM}^{9}\right.$ ) with 50 bootstrapping trials to estimate uncertainty using every 10 th frame. The statistical uncertainty was less than $0.1 \mathrm{kcal} / \mathrm{mol}$ for each data point.

\section{Unrestricted DFT/MM potential energy scans in singlet and triplet states}

A detailed description of the methods used for DFT/MM potential energy scans is included in the main text Methods with additional calculations described here. Besides using the unrestricted generalized gradient approximate (GGA) functional $\mathrm{PBE}^{10}$, the QM region was also modeled with BLYP ${ }^{1-12}$ and BP86 ${ }^{13}$ (Table S1). Other aspects of these DFT/MM calculations were unchanged. In order to check for any hysteresis in the forward potential energy scan, the model was driven from the products back to the reactants using the PBE/MM functional, which 
was followed by geometry optimization with the B3LYP/MM method. This scan was initiated from the most favorable structure of the product complex, but was otherwise performed in the same manner as the initial scan (Figure S4).

The potential energy profiles of the modified flavins were performed in the same manner as the native flavin. The initial structures were generated by replacing the C8 methyl group with the substituent of interest in the initial unrestricted B3LYP-minimized Michaelis complex structure (on the singlet surface) and the triplet structure at the corresponding reaction coordinate value. The dimethylamino-flavin geometry optimization was performed on the isoalloxazine ring of the flavin using the same DFT method described in the main text for the B3LYP/MM calculations.

\section{Spin flip}

The Restricted-Active-Space n-Spin Flip method with the double-zeta, polarized 6-31G* basis was used to characterize the electronic structures of the structures at reaction coordinates equal to $-0.2,0.05$, and $0.25 \AA$. This method starts from a high-spin reference state and correlates all electrons within singly occupied molecular orbitals (SOMO) at a complete-active-space (CAS) level, which captures the full effect of electronic correlations among the electrons in the frontier orbitals. Additional single excitations of the hole, particle type are used to capture the effects of orbital relaxations from this reference. Taken together, the RAS(h,p)-nSF level of theory captures multi-reference correlation effects at relatively low cost. Importantly, RAS-SF has been shown to consistently predict the correct spin state orderings for challenging molecular species with radical character, even for strongly correlated systems.

For all complexes, a single spin flip from a triplet reference structure was used. A second set of computations with an enlarged active space ( 4 electrons in 4 orbitals) was used to verify that the results did not qualitatively change with active space representation. These dependencies are

usually not observed, as supported by previous studies with RAS-SF ${ }^{14-16}$. Natural orbitals were constructed by diagonalizing the 1-particle density matrix on a state-specific basis.

\section{Visualization}

Spin-density images were generated in VMD (version 1.9.2) from OrcaPlot cube files. The isosurfaces were set to $+/-0.01$ for visualization. 


\section{DFTB3/MM free energy simulations}

Umbrella sampling was performed using the DFTB3/MM hybrid potential along the previously defined reaction coordinate (the mass-weighted distance difference between the peroxy $\mathrm{O} \delta$-Op bond and the forming $\mathrm{O} \delta$ - $\mathrm{C} 3$ bond [Reaction coordinate $=0.571(\mathrm{O} \delta$-Op-distance $)-0.429$ (C3-O 8 -distance)]). Thirty-three umbrella sampling windows were used with spring constants ranging from $50-250 \mathrm{kcal} \mathrm{mol}^{-1} \AA^{-2}$ to enhance sampling. Each window was simulated for $50 \mathrm{ps}$ with a 1 fs timestep using a Langevin thermostat (fbeta =5) at 298K. Analysis was performed on the last 40 ps of each simulation using the weighted histogram analysis method (WHAM ${ }^{9}$ ) with 50 bootstrapping trials to estimate uncertainty using every 10th frame. The statistical uncertainty was less than $0.1 \mathrm{kcal} / \mathrm{mol}$ for each data point.

The free energy barrier calculated from the DFTB3/MM simulations is significantly lower than the experimental hydroxylation free energy barrier measured with $\mathrm{PHBH}(15.0 \mathrm{kcal} / \mathrm{mol})$ and thus the accuracy of the model for determining transition state properties is uncertain. In addition, DFTB3 has not been extensively parameterized to accurately describe homolytic cleavage of the hydroperoxy $\mathrm{O}-\mathrm{O}$ bond and thus cannot be used to make distinctions between the competing mechanisms. Nevertheless, and as stated in the main text, the Michaelis complex and product structures are modeled in the correct electronic state and thus were analyzed further. 


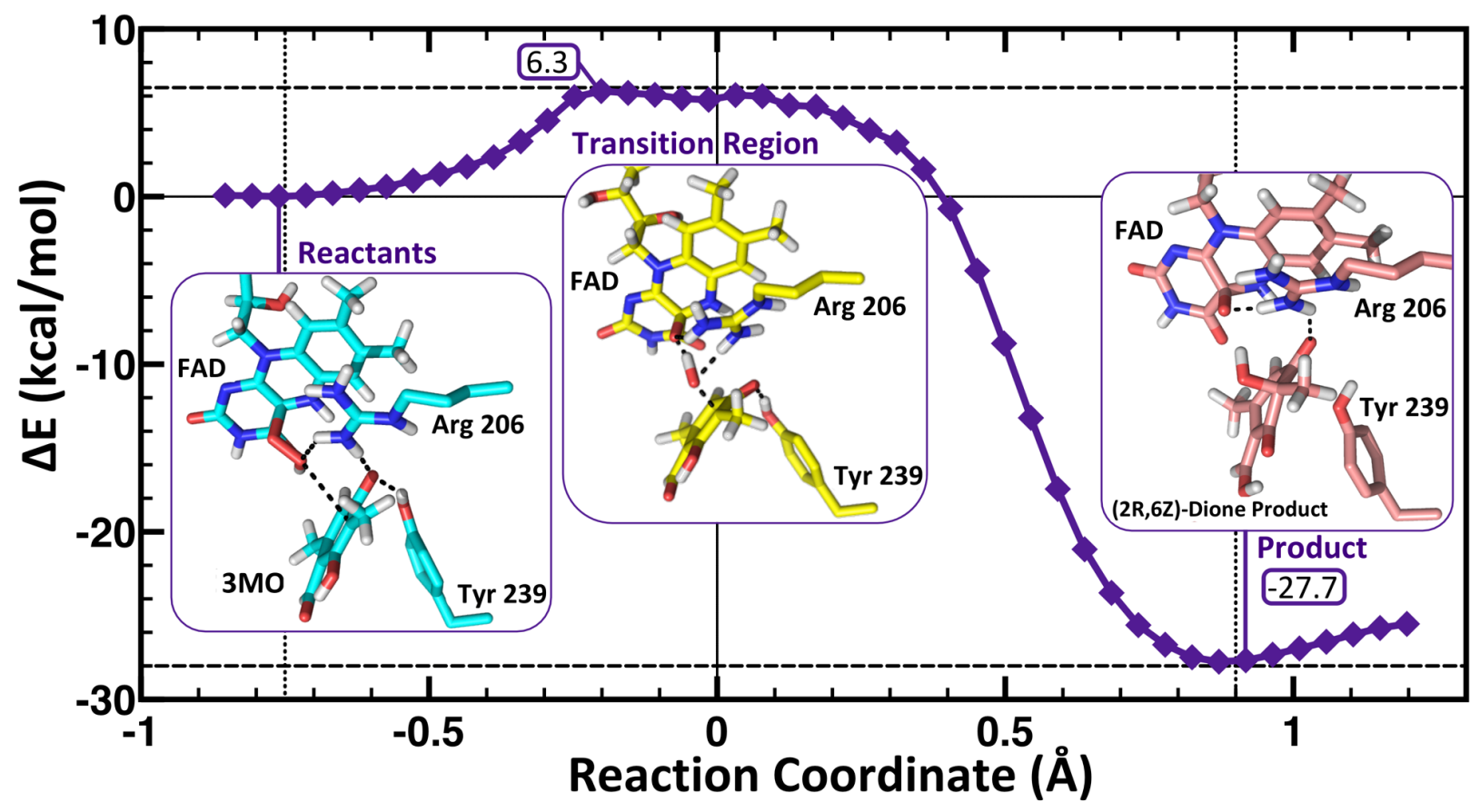

Figure S1: DFTB3/MM singlet free energy profile for hydroxylation of 3-methyl-orcinaldehyde from umbrella sampling and WHAM analysis. Representative structures from the Michaelis, transition, and product regions are shown in cyan, yellow and pink sticks respectively. The reaction coordinate used was the mass-weighted $(\mathrm{Op}-\mathrm{O} \delta)-(\mathrm{O} \delta-\mathrm{C} 3)$ distance difference. 

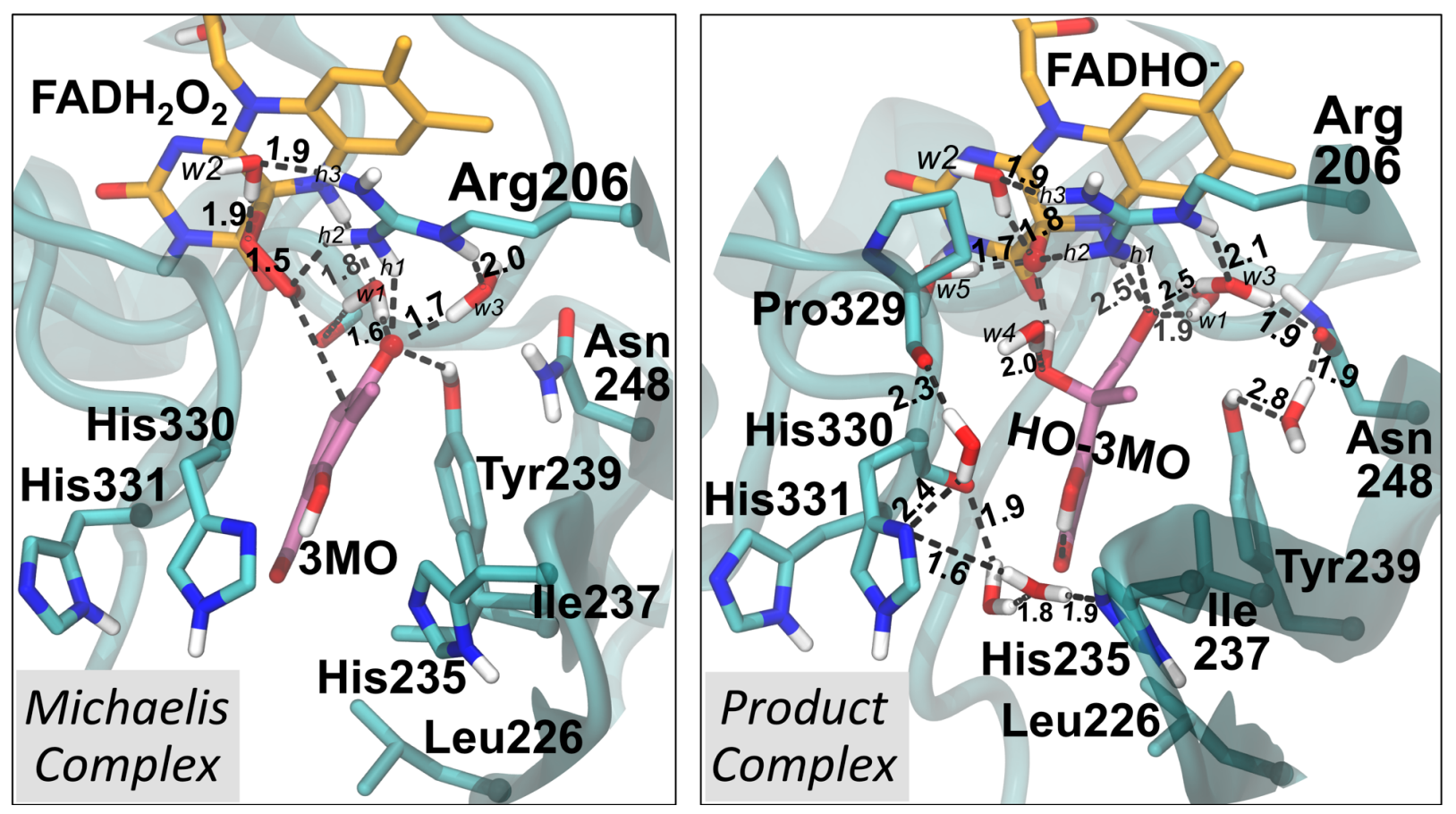

Figure S2: Representative structures from the A) Michaelis and B) product regions of the DFTB3/MM free energy profile. Distances not included in the main text Figure 2 are labeled with average values in Angstroms. Water molecules and hydrogens of R206 that are referred to in the Supplementary Tables (S1 and S4-S6) are labeled in italics. The flavin is shown in golden sticks, the substrate is shown in pink sticks, the amino acid side chains are shown in cyan sticks, the protein backbone is shown in transparent cyan cartoon, and active site water molecules are represented by sticks. The reaction coordinate used was the mass-weighted $(\mathrm{Op}-\mathrm{O} \delta)-(\mathrm{O} \delta-\mathrm{C} 3)$ distance difference. 
Table S1: Distances in Michaelis complex and product structures. Distances are averaged over the DFTB3/MM simulations near the Michaelis complex and product minima. For atom and molecule labels see Figure S2 and Table S4.

\begin{tabular}{|c|c|c|}
\hline Distances [Å] & $\begin{array}{l}\text { Michaelis } \\
\text { Complex }\end{array}$ & $\begin{array}{l}\text { Product } \\
\text { Complex }\end{array}$ \\
\hline OS-Op & 1.5 & \\
\hline O8-C3 & 3.8 & \\
\hline O8-Arg206H2 & 2.2 & \\
\hline Owat1-H(N5) & 1.8 & \\
\hline 04-Tyr239H & 1.9 & \\
\hline O4-Hwat1 & 1.6 & 1.9 \\
\hline 04-Arg206H1 & 2.0 & 2.3 \\
\hline 04-Hwat3 & 1.7 & 2.5 \\
\hline Owat3-Arg206HE & 2.0 & 2.1 \\
\hline Owat2-R206H3 & 1.9 & 1.9 \\
\hline Op-Hwat2 & 1.9 & 1.8 \\
\hline Op-Hwat5 & & 1.7 \\
\hline Op-Arg206H2 & & 1.8 \\
\hline Op-H(Oঠ) & & 1.7 \\
\hline 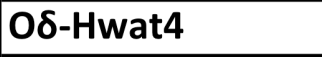 & & 2.0 \\
\hline O7-H(02) & & 1.5 \\
\hline
\end{tabular}

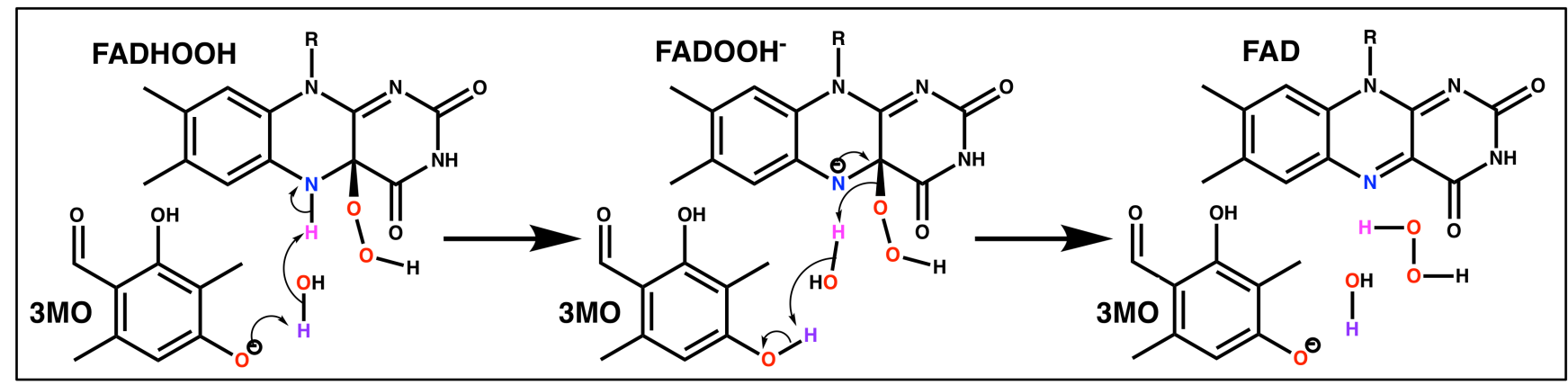

Figure S3: Scheme illustrating hydrogen peroxide formation during DFTB3/MM US simulations.

For discussion of the detailed mechanism of $\mathrm{H}_{2} \mathrm{O}_{2}$ formation see the main text. 


\section{Multiple sequence alignment of 280 fungal flavin-dependent hydroxylases}

A PSI-BLAST ${ }^{17}$ search was performed using the NCBI non-redundant protein database and the TropB sequence as the seed. Three rounds of searching were performed and 500 sequences were ultimately extracted from the database. The program $\mathrm{CD}-\mathrm{HIT}^{18}$ was used to remove fragments and sequences sharing more than $90 \%$ sequence identity. The sequences were aligned using MUSCLE ${ }^{19}$ and visualized using Genedoc. The MEME program ${ }^{20}$ was used to determine the 20 most conserved patterns/motifs in the sequence set. Additional sequences were deleted that were missing highly conserved motifs resulting in a total of 278 sequences. Small manual adjustments were made to the multiple sequence alignment such that all highly conserved motifs were fully aligned.

\section{B3LYP/MM potential energy scans checking for hysteresis}

\section{Evaluation of Hysteresis}

In order to check for any hysteresis in the forward potential energy scan performed with PBE/MM hybrid potential and minimized at the B3LYP/MM level, the model was driven from the products back to the reactants initiated from the most favorable structure of the product complex (Figure S4).

Driving the reaction from the product structure back towards the Michaelis complex produced a very similar profile to the initial results, suggesting that the profile was minimally biased by hysteresis (Figure S4). Additional forward driving, however, characterized a more defined and favorable product basin with the hydroperoxy-proton positioned primarily on the hydroxyflavin, and the anionic product hydrogen bonding with R206 at two positions. The product structure resulting from DFTB3/MM umbrella sampling simulations, however, is more consistent with the initial reaction coordinate driving, with the proton primarily on the product hydrogen bonding with the anionic alkoxyflavin. In addition, minimizing a structure from the middle of the (restricted) singlet transition region to the unrestricted triplet surface produced a structure that was $7.3 \mathrm{kcal} / \mathrm{mol}$ more favorable than minimizing the corresponding triplet structure to the restricted singlet surface. In addition, these points are similar in energy (Figure S4) to the structures generated through reaction coordinate driving along the respective surfaces, suggesting that 
structural differences between the surfaces are relatively minor and the relative energy of the two states is consistent. These calculations used 40 steps of further B3LYP/MM minimization, starting from the B3LYP/MM minimized structures from the initial reaction coordinate driving.

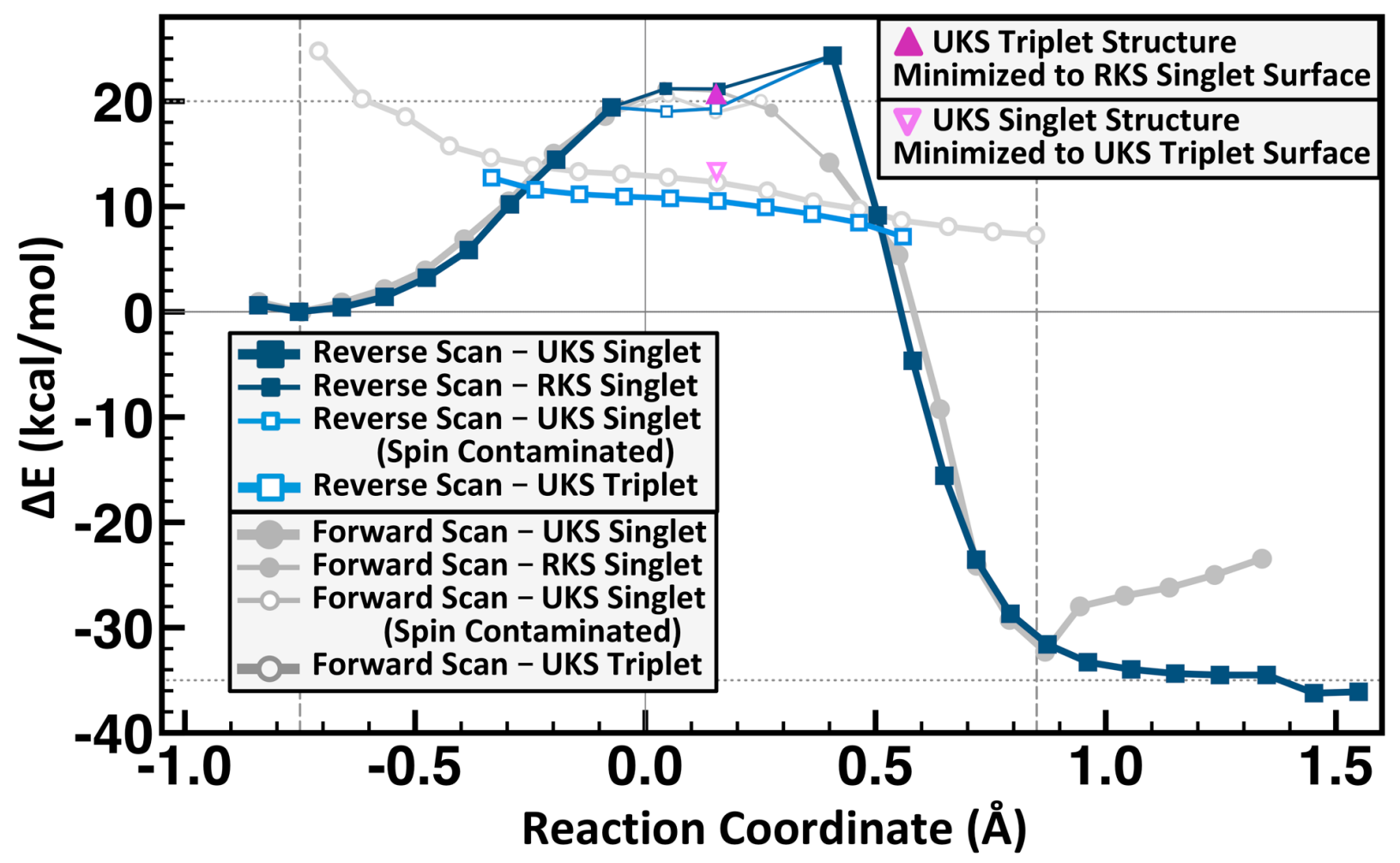

Figure S4: B3LYP/MM potential energy profile for hydroxylation of 3-methyl-orcinaldehyde when reaction coordinate driving was performed starting from the Michaelis complex ("Forward Scan") and starting from the product complex ("Reverse Scan"). In addition, structures from the middle of the singlet and triplet surfaces were minimized to the other surface (triplet and singlet respectively). The restricted/unrestricted calculations are adjusted based on the energy of the Michaelis complex calculated with restricted/unrestricted methods respectively. The reaction coordinate used was the mass-weighted $(\mathrm{Op}-\mathrm{O} \delta)-(\mathrm{O} \delta-\mathrm{C} 3)$ distance difference. 


\section{Comparison of DFT/MM methods}

\section{Comparison of QM Models}

Potential energy scans in the singlet and triplet states were first performed with several generalized gradient approximated (GGAs) density functionals including PBE, BLYP and BP86. All of these methods gave small singlet-triplet gaps (Table S2). Since it is well-established that GGAs differentially favor low spin states over higher ones ${ }^{21}$, the more accurate B3LYP method that includes Hartree-Fock exchange was used. The singlet-triplet gap calculated with this method was further examined with the DLPNO-CCSD(T)/MM method and complete active space (CAS) calculations (Section VI).

Table S2: Values for reaction barriers, singlet-triplet gaps, and energy changes are shown for the different QM/MM models used.

\begin{tabular}{|c|c|c|c|c|c|c|c|c|}
\hline $\begin{array}{c}\text { Energy } \\
{[\mathrm{kcal} / \mathrm{mol}]}\end{array}$ & $\begin{array}{c}\text { B3LYP } \\
\text { (Reverse } \\
\text { Scan) }\end{array}$ & $\begin{array}{c}\text { B3LYP } \\
\text { (Forward } \\
\text { Scan) }\end{array}$ & $\begin{array}{c}\text { PBE } \\
\text { (Reverse } \\
\text { Scan) }\end{array}$ & $\begin{array}{c}\text { PBE } \\
\text { (Forward } \\
\text { Scan) }\end{array}$ & $\begin{array}{c}\text { BLYP } \\
\text { (Forward } \\
\text { Scan) }\end{array}$ & $\begin{array}{c}\text { BP86 } \\
\text { (Forward } \\
\text { Scan) }\end{array}$ & $\begin{array}{l}\text { DFTB3 } \\
\text { US }^{+} \\
\text {Profile }\end{array}$ & $\begin{array}{l}\text { DFTB3 } \\
\text { RXNC }^{\#} \\
\text { Profile }\end{array}$ \\
\hline $\begin{array}{c}\Delta \mathrm{E} \text { Reactants to } \\
\text { Products }\end{array}$ & -36.2 & -32.3 & -29.8 & -31.3 & -26.4 & -30.8 & -27.7 & -36.7 \\
\hline Singlet Barrier & 21.2 & 21.2 & 11.8 & 8.5 & 4.9 & 8.5 & 6.3 & 15.1 \\
\hline Triplet Barrier & 11.6 & 13.8 & 9.1 & 8.8 & 4.1 & 8.2 & & \\
\hline $\begin{array}{c}\text { Singlet-Triplet } \\
\text { Barrier Diff. }\end{array}$ & 9.6 & 7.4 & 2.6 & -0.3 & 0.8 & 0.3 & & \\
\hline $\begin{array}{c}\text { Singlet-Triplet } \\
\text { Gap* }\end{array}$ & 8.8 & 8.6 & 3.9 & -0.3 & 1.7 & 0.8 & & \\
\hline
\end{tabular}

* Largest singlet-triplet energy difference between UKS calculations at similar reaction coordinates

+ Umbrella sampling (US)

\# Reaction coordinate driving (RXNC) 


\section{Restricted-Active-Space n-Spin Flip results}

\section{Molecular orbital analysis of 3-methyl-orcinaldehyde hydroxylation}

The oxidative character of the reaction is reflected in the charges of the flavin and substrate, as well as in the molecular orbitals before and after the elementary step. Prior to reaction, the highest occupied molecular orbital is a $\pi$ orbital on the substrate, and the lowest unoccupied molecular orbital (LUMO) resides on the hydroperoxy cofactor as a second (empty) $\pi$ orbital (Figure S5). The substrate has a net negative charge (-0.85) due to being deprotonated. After reaction, the oxidization of the substrate is evident by the transfer of $0.6 \mathrm{e}$ - to the flavin, leaving the product with $-0.24 \mathrm{e}$ - of charge. This change in charge comes with an exchange in location of the HOMO and LUMO, with the HOMO now residing on the flavin and the LUMO on the product (all orbitals are $\pi$ orbitals). This elementary step, however, proceeds through an unexpected change in electronic state along the reaction path.

The geometries from the DFT/MM calculation that pass from reactant to product reveal significant biradical character near the transition state region. Further strengthening this conclusion, high-level wave-function calculations indicate that just prior to the transition state at geometry point 1 the HOMO and LUMO are bonding and antibonding combinations of a flavin $\mathrm{OOH} \sigma$ orbital and the substrate's highest $\pi$ orbital (Figure S5). This geometry has begun to build up diradical character and the singlet is the lowest energy state, but with a relatively small tripletsinglet gap (Table S2). This radical character exists due to the partial breaking of the $\mathrm{O}-\mathrm{OH}$ bond in the peroxy group as the $\mathrm{OH}$ approaches the substrate. At the transition geometry, the electronic structure becomes fully diradical, with both the singlet or triplet state having two unpaired electrons. One electron resides in the flavin $\pi$ system, the second on the reacting $\mathrm{OH}$ group (the two orbitals representing this diradical are simple $+/$ - combinations of flavin and $\mathrm{OH}$ orbitals). Just after the transition state at geometry point 5 , the (doubly occupied) HOMO resides on the flavin, while the LUMO is located on the now oxidized substrate.

Since RAS-SF reports that the lowest-lying triplet and singlet states are diradicals at the transition state geometry, these simulations add evidence that closed-shell singlet pathways will be disfavored compared to the radical transition state. From an electronic structure perspective, the triplet state will always be open shell, and therefore easily described by a single reference

configuration. The quantum mechanically correct description of the open-shell singlet, on the other 
hand, requires a multi-reference description at the transition state to provide the correct spin expectation value (i.e. $\mathrm{S}^{2}=0$ ). This wave function method therefore differs from methods like DFT, where an open-shell singlet has artificial values of the $S^{2}$ expectation value.

This overall transformation can be succinctly described as a radical transition state for oxidation, resulting in overall net electron transfer to the flavin. The oxidation and $\mathrm{OH}$ transfer occur in the middle of this reactive sequence, and occur by an adiabatic switch in electronic state from closed shell singlet to diradical and back to closed shell singlet, all along a single elementary step energetic profile. 

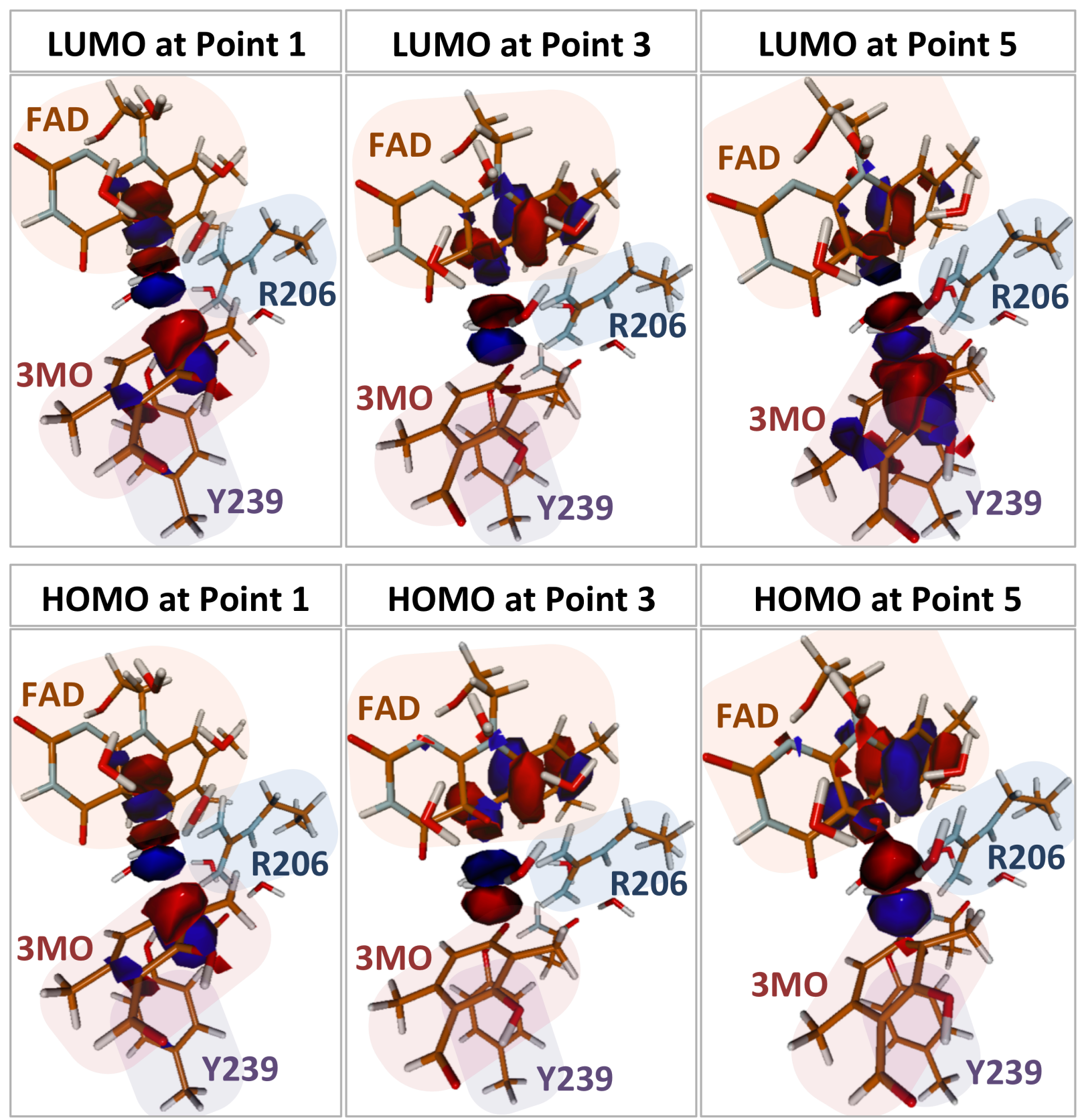

Figure S5: Highest-occupied and lowest-unoccupied molecular orbitals from the RAS-SF calculations are shown for 3 points on the singlet surface. Corresponding singlet surface numbering and orbital occupancies are shown in Table S3. Positive and negative orbitals are shown in red and blue respectively while the structures are shown in sticks. The flavin is highlighted in orange, the substrate is highlighted in pink, Arg206 is highlighted in blue and Tyr239 is highlighted in purple. 
Table S3. Orbital occupancies near the transition state region according to RAS-SF (top) and transition region of the B3LYP energy profile labeled with points corresponding to singlet structures used for RAS-SF calculations (bottom). The plot below the table is reproduced from Figure $\mathrm{S} 4$ of the main text for easy reference.

\begin{tabular}{|cc|c|c|c|}
\hline \multirow{2}{*}{ Orbital Occupancies } & \multicolumn{3}{|c|}{ Geometries } \\
\cline { 3 - 6 } & Point 1 & Point 3 & Point 5 \\
\hline \multirow{2}{*}{ Singlet } & LUMO & 0.37 & 0.93 & 0.29 \\
\cline { 2 - 5 } & HOMO & 1.63 & 1.07 & 1.80 \\
\hline \multirow{2}{*}{ Triplet } & LUMO & 1 & 1 & 1 \\
\cline { 2 - 5 } & HOMO & 1 & 1 & 1 \\
\hline
\end{tabular}

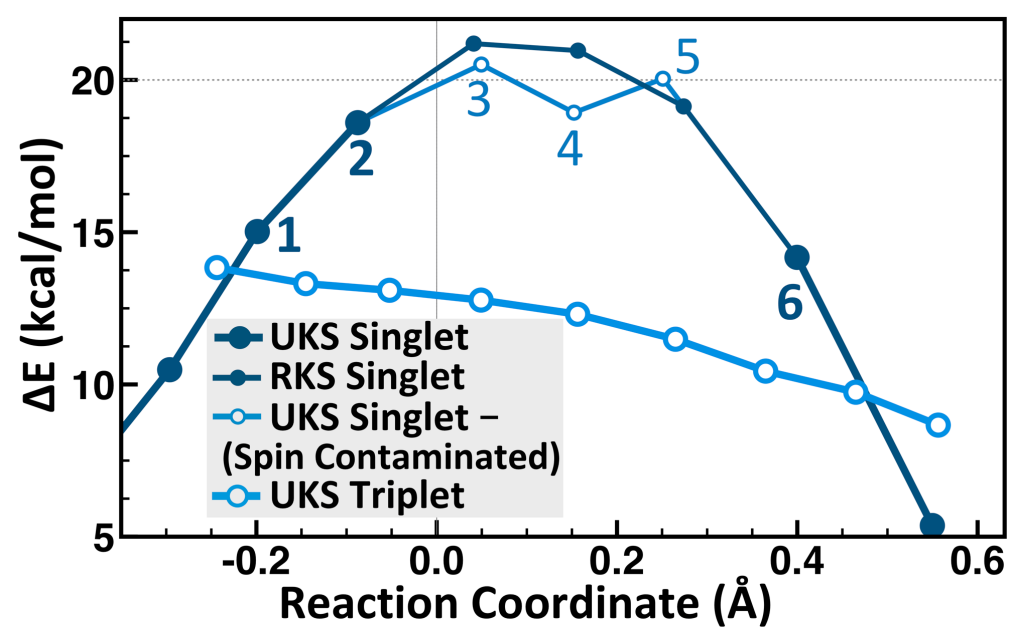




\section{Detailed analysis of mechanism from B3LYP/MM method}

\section{Hydroxylation of 3-methyl-orcinaldehyde}

Potential energy scans were performed on the singlet and triplet surfaces with the PBE/MM hybrid functional, using the previously defined reaction coordinate (the mass-weighted distance difference between the peroxy $\mathrm{O} \delta$-Op bond and the forming $\mathrm{O} \delta$ - $\mathrm{C} 3$ bond [Reaction coordinate $=$

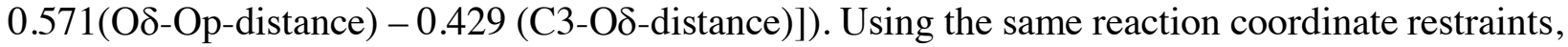
40 additional steps of geometry optimization were performed with the unrestricted B3LYP/MM functional. Additional charge and spin distribution analyses were performed with natural-bond orbital analysis $(\mathrm{NBO})^{22}$ through the Q-Chem/CHARMM interface ${ }^{23}$. These analyses can provide additional description of the reaction mechanism.

Detailed interrogation of the unrestricted B3LYP/MM reaction surface reveals that the biradical formation is initiated with Oठ-Op bond cleavage and after the system traverses the broad triplet transition region, $\mathrm{C} 3-\mathrm{O} \delta$ bond formation and electron transfer return the system to the

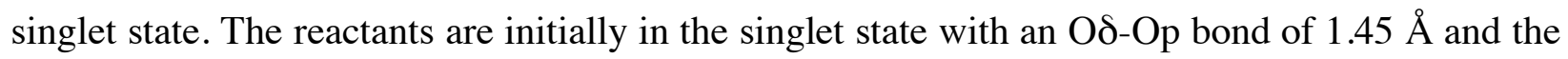
C3-Od distance of $3.7 \AA$ (Table S5). As the substrate is positioned closer to the reactive cofactor, the O\&-Op bond elongates to $2.1 \AA$ and the system crosses to the triplet state. Just before the crossing point on the singlet surface, the flavin ring has a small net positive charge of +0.29 while the hydroxyl group (Oס is neutral and the charge on Op is -0.34 (which is similar to the value of 0.42 predicted by Ortiz-Maldonado ${ }^{24}$ ) according to Natural Bond Orbital (NBO) analysis (Table S4). Near the initial crossing on the triplet surface (after O $\delta$-Op bond cleavage), however, the flavin ring transfers significant charge to the hydroxyl group and Op, resulting in a +0.8 charge on the ring and -0.6 charge on Op. This is the highest energy point on the triplet surface indicating that $\mathrm{C} 8$-substitutions to the flavin cofactor will influence OS-Op bond cleavage and the barrier height for the triplet mechanism. The highest energy point on the singlet surface, in contrast, is near the middle of the transition region, and shows maintenance of the initial flavin ring charge along with less charge on Op (-0.5e) and more charge on the substrate (-0.5e) than the highest energy structure on the triplet surface. As the system progresses through the triplet transition region, the charge on Op increases to -0.8 as the charge on the substrate decreases to -0.4 . The $\mathrm{C} 3-\mathrm{O} \delta \mathrm{H}$ distance decreases to $2.5 \AA$ forming a dative bond and the O $\delta$-Op distance increases to 2.7 Å before

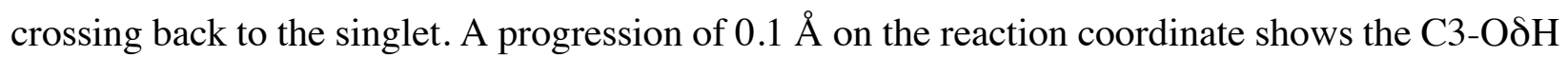
bond distance decrease dramatically on the singlet surface to $1.6 \AA$ and nearing the equilibrium 
value in the following step. Formation of the product leaves the alkoxyflavin with the majority of the anionic charge (-0.6).

The C4-O4 double bond is already partially formed in the Michaelis complex due to prior deprotonation of the substrate and is strengthened by the hydroxylation reaction. The C4-O bond is $1.31 \AA$ in the Michaelis complex, and decreases slightly in the triplet transition region before crossing to the singlet and decreasing further to $1.25 \AA$. The C3-C4 and C4-C5 $\pi$ bonds that are weakened by the deprotonation of the hydroxy group are $1.41 \AA$ and $1.42 \AA$ respectively in the Michaelis complex while the adjacent C3-C2 bond is not affected and remains at 1.37 $\mathrm{A}$. This C3$\mathrm{C} 4$ bond is further lengthened in the triplet transition region before reaching a typical single bond length of $1.52 \AA$ in the product structure. The O $\delta-\mathrm{H}$ proton hydrogen bonds with the nearby carbonyl oxygen atom of the cofactor (O4) in the Michaelis complex and through the transition region; this hydrogen bond is only broken once the product is formed.

Table S4: NBO spin density distribution in the transition state region of the B3LYP triplet reaction profile: average distribution within the flavin and substrate (top) and distribution throughout the transition state region (bottom).

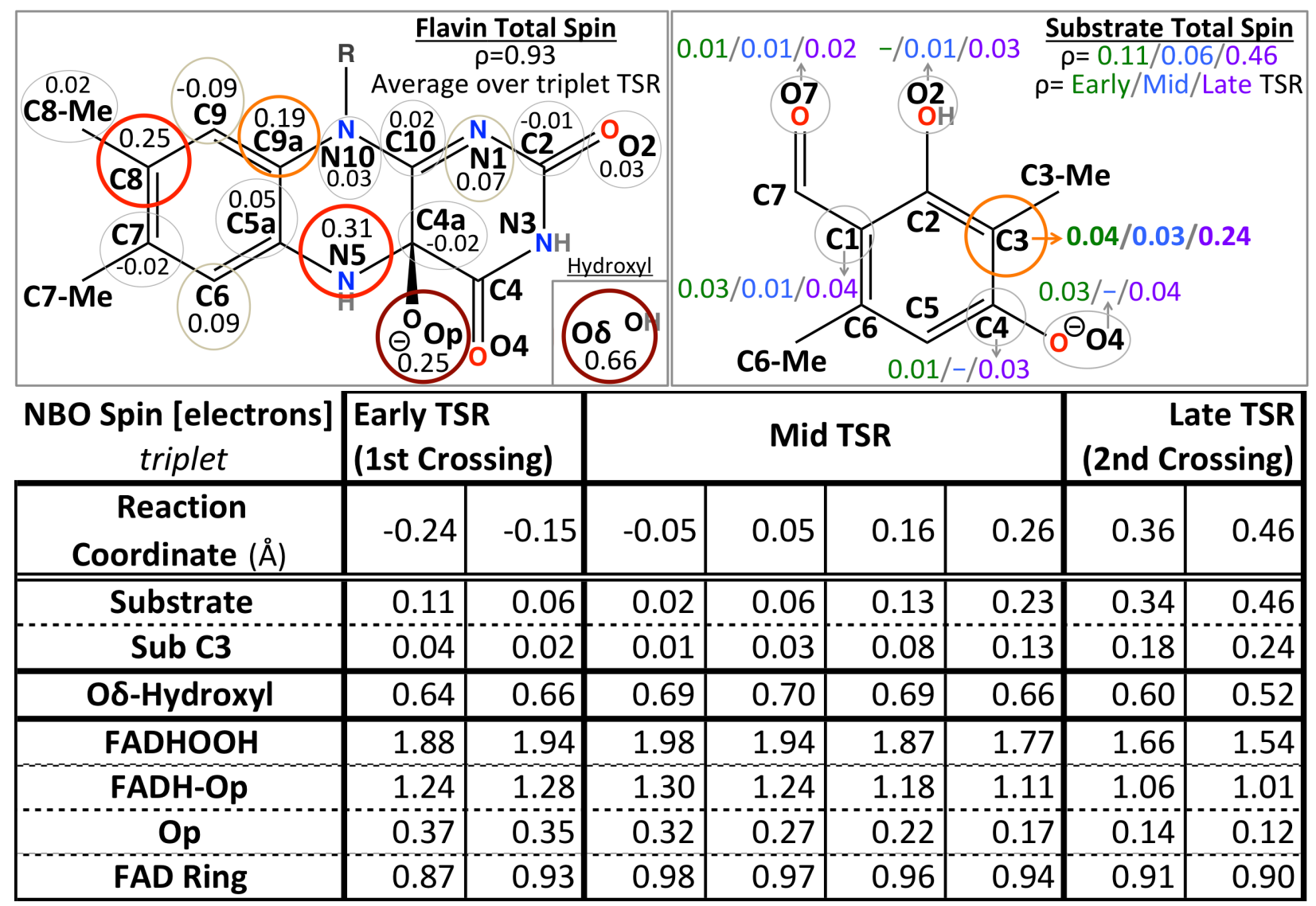


Table S5: NBO charge distribution along the B3LYP reaction profile.

\begin{tabular}{|c|c|c|c|c|c|c|}
\hline \multicolumn{2}{|l|}{ NBO Charges [e] } & $\begin{array}{l}\text { Michaelis } \\
\text { Complex }\end{array}$ & $\begin{array}{c}\text { Early TSR } \\
\text { (1st Crossing) }\end{array}$ & Mid TSR & \begin{tabular}{|c|} 
Late TSR \\
(2nd Crossing)
\end{tabular} & $\begin{array}{l}\text { Product } \\
\text { Complex }\end{array}$ \\
\hline \multirow{2}{*}{$\begin{array}{c}\text { Reaction } \\
\text { Coordinate }(\AA)\end{array}$} & singlet & -0.75 & -0.20 & 0.04 & 0.55 & 0.87 \\
\hline & triplet & & -0.24 & 0.05 & 0.46 & \\
\hline \multirow{2}{*}{ Substrate } & singlet & -0.85 & -0.77 & -0.52 & 0.20 & \\
\hline & triplet & & -0.74 & -0.77 & -0.42 & \\
\hline \multirow{2}{*}{ Product } & singlet & & & & -0.02 & -0.24 \\
\hline & triplet & & & & -0.76 & \\
\hline \multirow{2}{*}{ Substrate C3 } & singlet & -0.17 & -0.16 & -0.08 & 0.14 & 0.19 \\
\hline & triplet & & -0.14 & -0.14 & -0.01 & \\
\hline \multirow{2}{*}{ Oठ-Hydroxyl } & singlet & 0.00 & -0.03 & -0.13 & -0.22 & -0.39 \\
\hline & triplet & & -0.27 & -0.21 & -0.34 & \\
\hline \multirow{2}{*}{ FADHOOH } & singlet & -0.03 & -0.10 & & & \\
\hline & triplet & & -0.07 & & & \\
\hline \multirow{2}{*}{ FADH-Op } & singlet & & -0.07 & -0.23 & -0.83 & -0.60 \\
\hline & triplet & & 0.19 & 0.20 & 0.00 & \\
\hline \multirow{2}{*}{ FAD Ring } & singlet & 0.28 & 0.29 & 0.26 & 0.05 & 0.20 \\
\hline & triplet & & 0.83 & 0.90 & 0.80 & \\
\hline \multirow{2}{*}{ Op } & singlet & -0.31 & -0.36 & -0.49 & -0.88 & -0.80 \\
\hline & triplet & & -0.64 & -0.70 & -0.80 & \\
\hline
\end{tabular}


Table S6: Distances along the B3LYP reaction profile.

\begin{tabular}{|c|c|c|c|c|c|c|}
\hline Distances $[\AA]$ & & $\begin{array}{l}\text { Michaelis } \\
\text { Complex }\end{array}$ & $\begin{array}{c}\text { Early TSR } \\
\text { (1st Crossing) }\end{array}$ & Mid TSR & $\begin{array}{c}\text { Late TSR } \\
\text { (2nd Crossing) }\end{array}$ & $\begin{array}{l}\text { Product } \\
\text { Complex }\end{array}$ \\
\hline \multirow{2}{*}{$\begin{array}{c}\text { Reaction } \\
\text { Coordinate }\end{array}$} & singlet & -0.75 & -0.20 & 0.04 & 0.55 & 0.87 \\
\hline & triplet & & -0.24 & 0.05 & 0.46 & \\
\hline \multirow{2}{*}{ Oঠ-Op } & singlet & 1.45 & 1.65 & 1.89 & 2.18 & 2.58 \\
\hline & triplet & & 2.12 & 2.31 & 2.70 & \\
\hline \multirow{2}{*}{ Oঠ-C3 } & singlet & 3.67 & 2.66 & 2.42 & 1.62 & 1.41 \\
\hline & triplet & & 3.39 & 2.96 & 2.51 & \\
\hline \multirow{2}{*}{ O4-C4 } & singlet & 1.31 & 1.31 & 1.29 & 1.25 & 1.25 \\
\hline & triplet & & 1.30 & 1.31 & 1.29 & \\
\hline \multirow{2}{*}{ C3-C4 } & singlet & 1.41 & 1.41 & 1.44 & 1.51 & 1.52 \\
\hline & triplet & & 1.42 & 1.41 & 1.44 & \\
\hline \multirow{2}{*}{ 04-Tyr239H } & singlet & 1.73 & 1.67 & 1.70 & 1.73 & 1.79 \\
\hline & triplet & & 1.63 & 1.61 & 1.63 & \\
\hline \multirow{2}{*}{ 04-Hwat1 } & singlet & 1.71 & 1.67 & 1.72 & 1.87 & 1.77 \\
\hline & triplet & & 1.77 & 1.71 & 1.76 & \\
\hline \multirow{2}{*}{ 04-Arg206H1 } & singlet & 1.69 & 1.66 & 1.69 & 1.77 & 1.76 \\
\hline & triplet & & 1.72 & 1.68 & 1.71 & \\
\hline \multirow{2}{*}{ Oঠ-Arg206H2 } & singlet & 1.97 & 2.03 & 2.07 & 2.21 & 2.46 \\
\hline & triplet & & 1.75 & 1.81 & 1.82 & \\
\hline \multirow{2}{*}{ 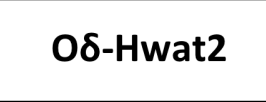 } & singlet & 2.23 & 1.99 & 1.84 & 1.76 & 1.54 \\
\hline & triplet & & 1.71 & 1.68 & 1.59 & \\
\hline
\end{tabular}


VIII. B3LYP/MM calculations with only the flavin and substrate represented in the QM region

Charge transfer from the substrate to the surroundings was observed in all of our QM/MM calculations. In order to determine the impact of this interaction on the potential energy profile, the QM region was reduced to just the substrate and the cofactor. In addition, all of the reported calculations on the hydroxylation reaction in $\mathrm{PHBH}$ models represent the QM region with just these two moieties ${ }^{25-29}$. The potential energy barrier is increased by $\sim 8 \mathrm{kcal} / \mathrm{mol}$ with a smaller QM region in the TropB model. Interestingly, the triplet surface does not cross the singlet surface and no spin contamination is obtained from the singlet calculations that could indicate an alternative mechanism. Thus, it would be interesting to reinvestigate the PHBH mechanism with unrestricted methods and the substrate in anionic and dianionic form.

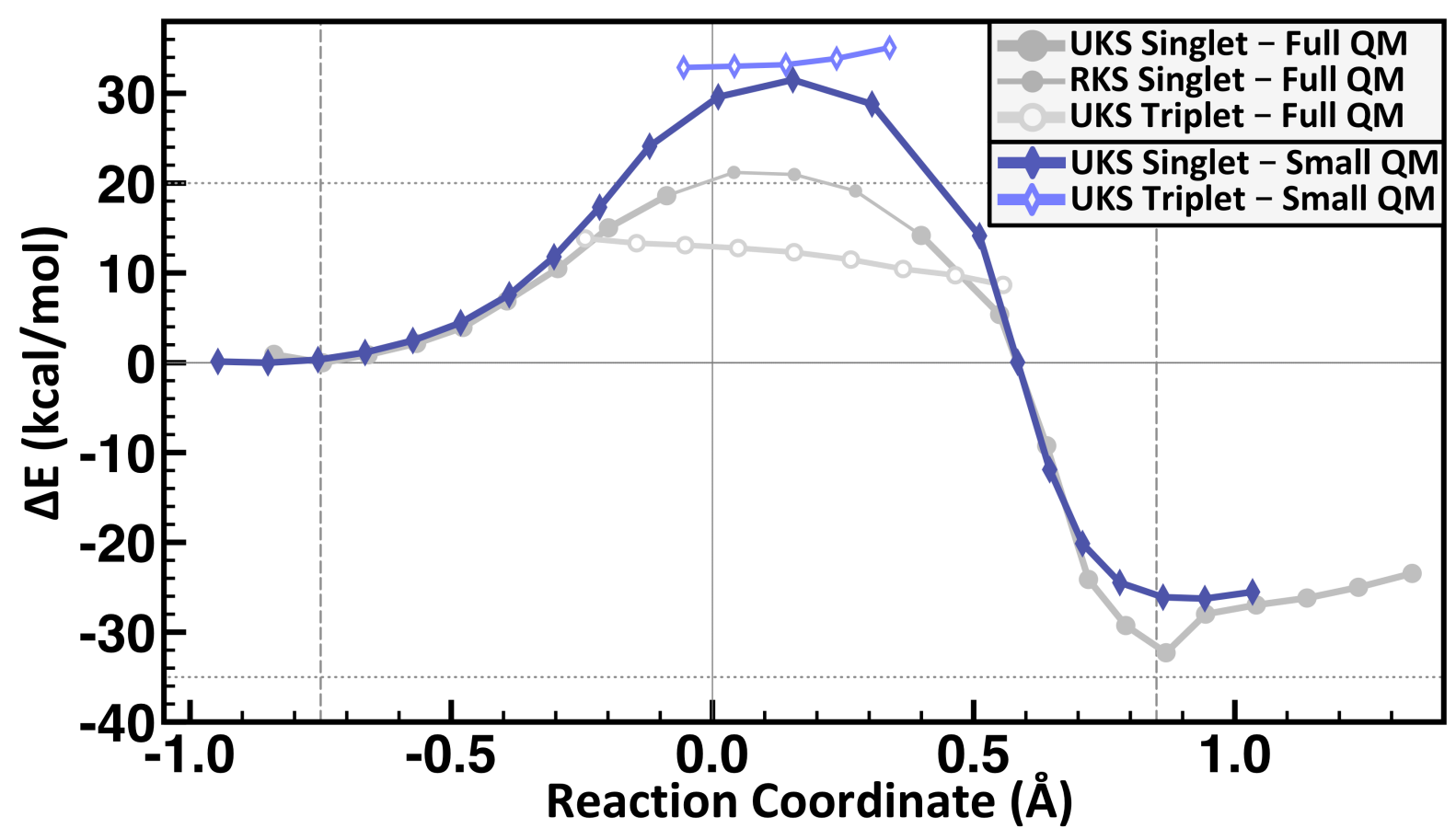

Figure S6: B3LYP/MM potential energy profile for hydroxylation of 3-methyl-orcinaldehyde with a minimal QM region consisting of the substrate and the flavin isoalloxazine ring. The singlet barrier height is $31.5 \mathrm{kcal} / \mathrm{mol}$ and the singlet-triplet gap is $1.7 \mathrm{kcal} / \mathrm{mol}$ favoring the singlet surface. These calculations followed the same methods as the original profile, but with the smaller QM region (reaction coordinate driving and minimization with $\mathrm{PBE}$ followed by 40 additional steps of minimization with unrestricted B3LYP). The reaction coordinate used was the mass-weighted $(\mathrm{Op}-\mathrm{O} \delta)-(\mathrm{O} \delta-\mathrm{C} 3)$ distance difference. 


\section{B3LYP/MM potential energy scans of the hydroxylation reaction with C8-modified flavins}

\section{The hydroxylation reaction with C8-substituted flavins}

In order to investigate the effect of flavin modifications on the biradical (HRC-ET) mechanism compared to the electrophilic mechanism, DFT/MM calculations were performed with C8-modified flavins in the TropB model. The substitutions were chosen to correspond with flavins experimentally investigated in $\mathrm{PHBH}^{24}$. The potential energy profiles of the modified flavins were performed in the same manner as the native flavin. The initial structures were generated by replacing the $\mathrm{C} 8$ methyl group with the substituent of interest in the initial unrestricted B3LYPminimized Michaelis complex structure (on the singlet surface) and the triplet structure at the corresponding reaction coordinate value. The dimethylamino-flavin geometry optimization was performed on the isoalloxazine ring of the flavin using the same DFT method described for the B3LYP/MM calculations. The results further favor the HRC-ET mechanism and demonstrate the impact of flavin substituents on the barrier of the triplet mechanism. A more detailed analysis of each substitution is presented below.

The $\mathbf{H}$ substitution is slightly less electron donating than the methyl group and has a slightly smaller rate constant in PHBH. The UB3LYP reaction profile in TropB closely mimics that of the native flavin, but both the singlet and triplet surfaces are about $1 \mathrm{kcal} / \mathrm{mol}$ higher in energy throughout the transition region. The triplet surface is highest in energy near the initial crossing, with a barrier height of $14.6 \mathrm{kcal} / \mathrm{mol}$, which is $0.8 \mathrm{kcal} / \mathrm{mol}$ higher than the native flavin (see Figure S7 and Table S6). The reaction is favorable by $27.6 \mathrm{kcal} / \mathrm{mol}$. The spin density is shifted compared to the native model with less spin on the flavin ring and more on the substrate ( $\rho=0.57$ vs. $0.9, \rho=0.42$ vs. 0.1 , respectively). Both surfaces qualitatively reproduce the slower hydroxylation rate of $-\mathrm{H}$ compared to the methyl group.

The Cl substitution has the fastest observed hydroxylation reaction rate among the substituted flavins in $\mathrm{PHBH}$, although the exact rate is obscured somewhat by the slower preceding step. This substituent is both resonance electron donating and inductively electron withdrawing. The profile in TropB closely mimics the reaction profile of the native flavin, but in the transition region both the singlet and triplet surfaces are slightly higher in energy. The triplet barrier height is $14.2 \mathrm{kcal} / \mathrm{mol}$, which is $0.4 \mathrm{kcal} / \mathrm{mol}$ higher than the native flavin, while the singlet barrier height is $21.9 \mathrm{kcal} / \mathrm{mol}$, which is $0.7 \mathrm{kcal} / \mathrm{mol}$ higher than the native flavin. The reaction is favorable by 
$-28.2 \mathrm{kcal} / \mathrm{mol}$. The distribution of spin closely corresponds with the values seen for the $-\mathrm{H}$ substitution. The triplet transition structure remains lower in energy than the singlet transition state structure by more than $7 \mathrm{kcal} / \mathrm{mol}$. The discrepancy between our calculated barrier and the faster rate for this substitution most likely arises from not including the effect of surface crossing contributions such as the well-established heavy atom effect that would certainly enhance the rate of reaction ${ }^{30}$. Thus, the faster rate for the $\mathrm{C} 8-\mathrm{Cl}$ substitution is most likely due to these contributions.

The $\mathbf{N H}_{2}$ substitution increases the reaction rate in $\mathrm{PHBH}$ but also has a significant decoupling component (15\%). $\mathrm{NH}_{2}$ is resonance electron donating. The crossing point from singlet to triplet in the TropB model is much earlier than the native flavin model at a reaction coordinate value of -0.45 resulting in a much smaller barrier of $4.4 \mathrm{kcal} / \mathrm{mol}$ (Figure S8). The overall energy change is $-25.1 \mathrm{kcal} / \mathrm{mol}$. Restricted singlet calculations show a barrier height of $20.8 \mathrm{kcal} / \mathrm{mol}$, which is lower than the spin-restricted singlet barrier for the native flavin by $0.4 \mathrm{kcal} / \mathrm{mol}$ but higher in energy than the amine triplet surface by $20 \mathrm{kcal} / \mathrm{mol}$. The unpaired electron on the cofactor is delocalized as in the native cofactor but extends beyond $\mathrm{C} 8$ to include the nitrogen atom bonded to $\mathrm{C} 8$. The $\mathrm{NH}_{2}$ substitution stabilizes the cationic isoalloxazine radical (by donating electron density from the lone pair) and thus there is donation of charge from the flavin ring to the proximal oxygen. Before crossing back to the singlet surface, the spin density on the substrate exhibits the same pattern as seen with the native cofactor. These calculations do not consider the effect of fluctuations that most likely serve to reduce the resonance effect of the $\mathrm{NH}_{2}$ group with the rings of $\mathrm{FAD}$. Thus, while the effect of placing a resonance electron donor at $\mathrm{C} 8$ is qualitatively reproduced, the magnitude of this substitution is most likely overestimated. In principle, this physical explanation could be tested by doing the experiments at varying temperatures.

The $\mathbf{N}-\left(\mathbf{C H}_{3}\right)_{2}$ dimethylamino substituent should have similar electronic qualities to $\mathrm{NH}_{2}$, but with a much greater steric footprint. To minimize the steric clash of the methyl groups with the adjacent 7-methyl substituent, the group must rotate to place one methyl above the plane of the flavin and one below (Figure S9). This rotation would prevent the overlap of the nitrogen-based lone pair with the $\pi$ system of the flavin. If the lone pair has minimal overlap with the $\pi$ system, then the inductive electron withdrawing effects likely dominate the contribution over the resonance electron donation. Thus, this substitution would effectively act as a weak, inductive electron- 
withdrawing group, and not as a resonance electron donating group like $\mathrm{NH}_{2}$, explaining the $4.5 \mathrm{x}$ slower hydroxylation rate of the dimethlamino flavin in $\mathrm{PHBH}$.

The CN substitution is resonance electron withdrawing and slows down the reaction with $\mathrm{O}_{2}$ to such an extent that measuring the precise hydroxylation rate is not possible in $\mathrm{PHBH}$, though the rate is reported to be faster than with the native cofactor. The cyano substitution shows a crossing to the triplet state at a point in the reaction coordinate very similar to the native $\mathrm{FADHOOH}$, but the characterization of the transition state region is very different. The O $\delta$-Op bond breaks homolytically but the unpaired electrons are not delocalized over FAD towards C8. Instead, one unpaired electron is distributed almost equally across the two oxygen atoms while the other one is instantaneously delocalized over the substrate. The UB3LYP/MM singlet-triplet gap is $\sim 4.4 \mathrm{kcal} / \mathrm{mol}$ and the overall energy change is $-34.2 \mathrm{kcal} / \mathrm{mol}$. The calculations indicate that the hydroperoxy-substrate biradical pair in the transition state is less favorable energetically than the hydroperoxy-flavin biradical pair. In the native form, the hydroxyl radical required a closer approach to $\mathrm{C} 3$ and subsequently induced a smaller spin density component before forming a bond. The potential energy actually increases within the triplet transition state region forming an intermediate that calculations suggest may be detectable.

These modifications demonstrate the importance of kinetics, thermodynamics, and dynamics in this reaction. The barrier height is primarily affected in $\mathrm{NH}_{2}$ and $\mathrm{CN}$, although dynamics have a clear influence on the magnitude of these barrier changes. The interaction of complex factors must be considered, not just the propensity for electron donation or contribution to the conjugated pi orbitals. 

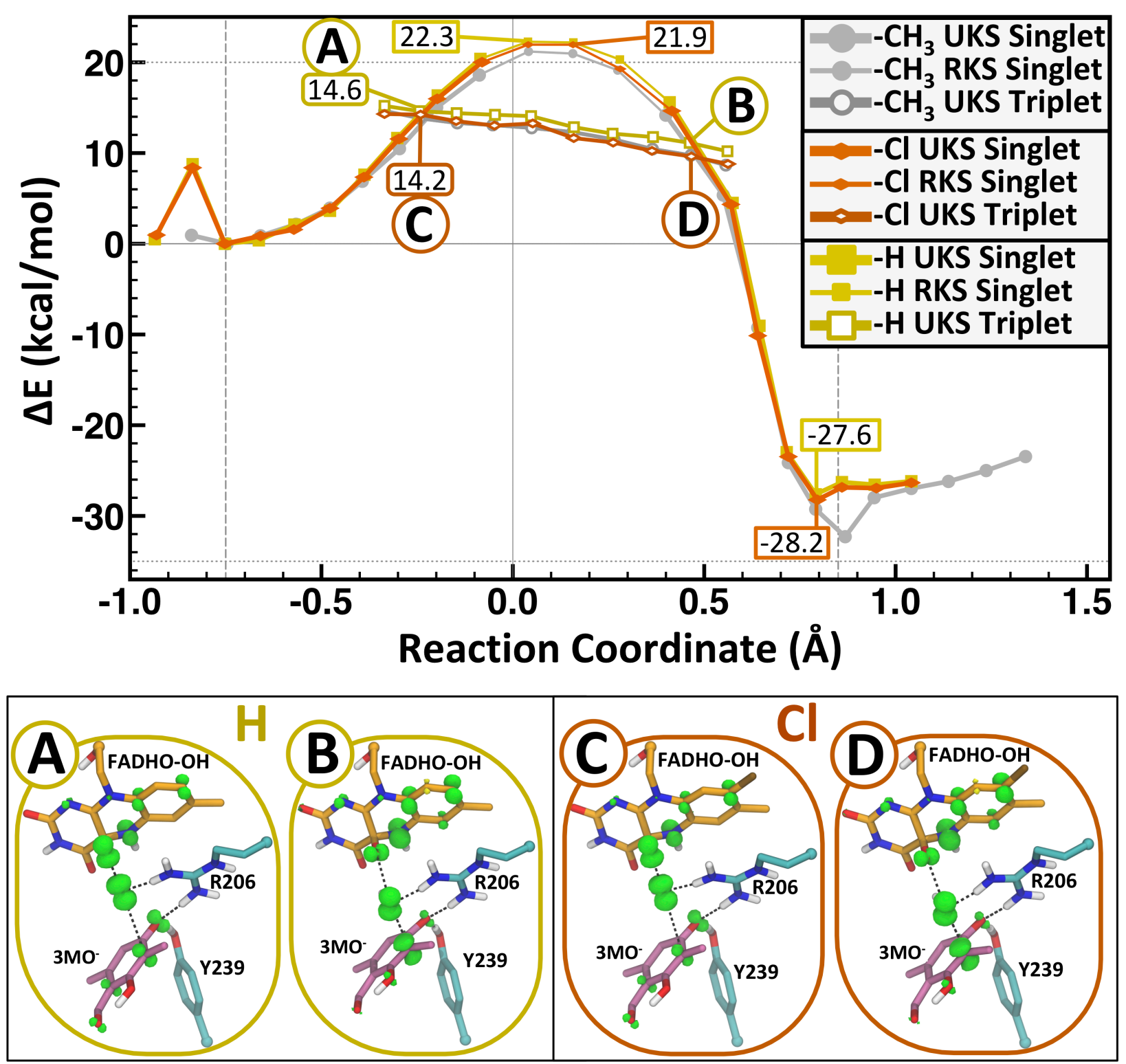

Figure S7: B3LYP/MM potential energy profile for hydroxylation of 3-methyl-orcinaldehyde with $\mathrm{C} 8-\mathrm{H}$ and $\mathrm{C} 8-\mathrm{Cl}$ modified flavins compared to the native flavin profile. Structures below illustrate the positive (green) and negative (yellow) spin density distribution at triplet points near the $1^{\text {st }}$ and $2^{\text {nd }}$ crossing points for $\mathrm{C} 8-\mathrm{H}(\mathrm{A} / \mathrm{B})$ and $\mathrm{C} 8-\mathrm{Cl}(\mathrm{C} / \mathrm{D})$. The flavin is represented in golden sticks, the amino acid side chains are represented in cyan sticks, and the substrate is shown in pink sticks. The reaction coordinate used was the mass-weighted $(\mathrm{Op}-\mathrm{O} \delta)-(\mathrm{O} \delta$-C3) distance difference. 

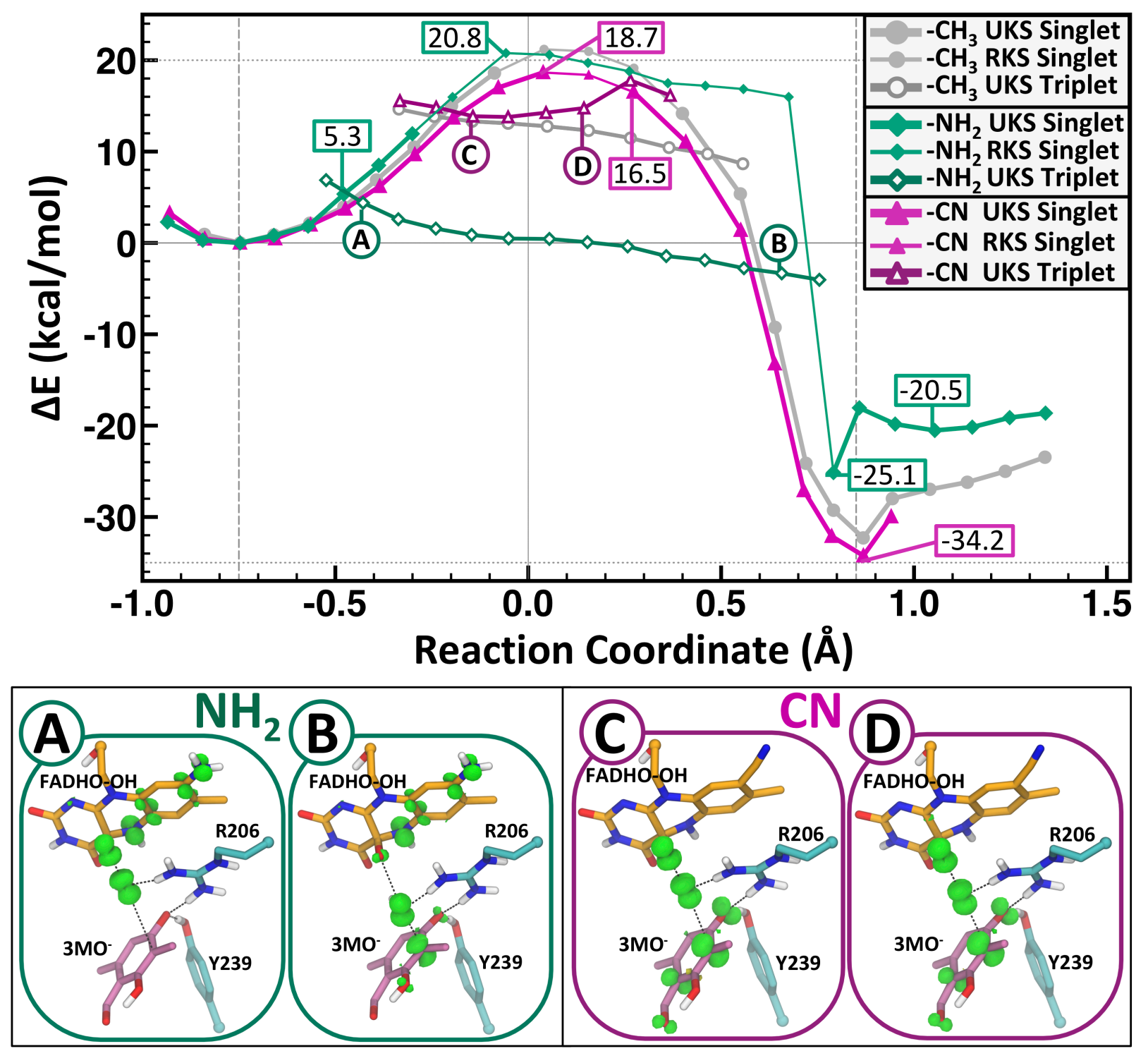

Figure S8: B3LYP/MM potential energy profile for hydroxylation of 3-methyl-orcinaldehyde with $\mathrm{C} 8-\mathrm{NH} 2$ and $\mathrm{C} 8-\mathrm{CN}$ modified flavins compared to the native flavin profile. Structures below illustrate the positive (green) and negative (yellow) spin density distribution at triplet points near the $1^{\text {st }}$ and $2^{\text {nd }}$ crossing points for $\mathrm{C} 8-\mathrm{NH}_{2}(\mathrm{~A} / \mathrm{B})$ and $\mathrm{C} 8-\mathrm{CN}(\mathrm{C} / \mathrm{D})$. The flavin is represented in golden sticks, the amino acid side chains are represented in cyan sticks, and the substrate is shown in pink sticks. The reaction coordinate used was the mass-weighted $(\mathrm{Op}-\mathrm{O} \delta)-(\mathrm{O} \delta-\mathrm{C} 3)$ distance difference. 


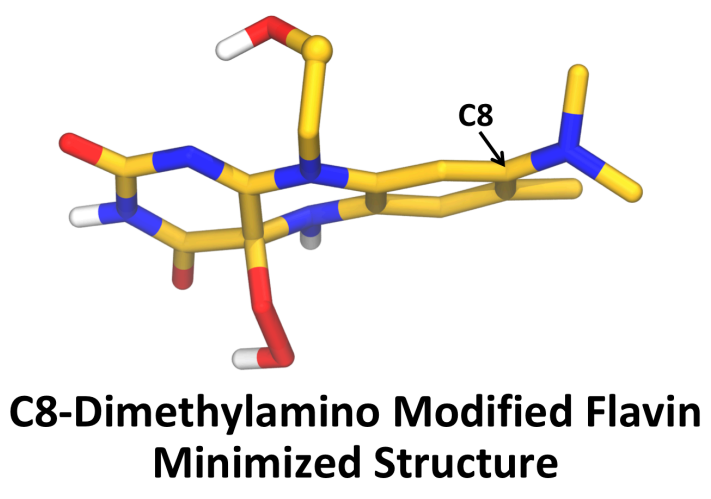

Figure S9: B3LYP-minimized structure for the C8-dimethylamino modified flavin. The substituent rotates to reduce steric clashes with the methyl groups, placing one group above the plane of the isoalloxazine ring and one group below. This results in the lone-pair of the nitrogen being oriented perpendicular to the plane of the flavin and its conjugated $\pi$ system.

Table S7: Summary values for modeled modified flavins.

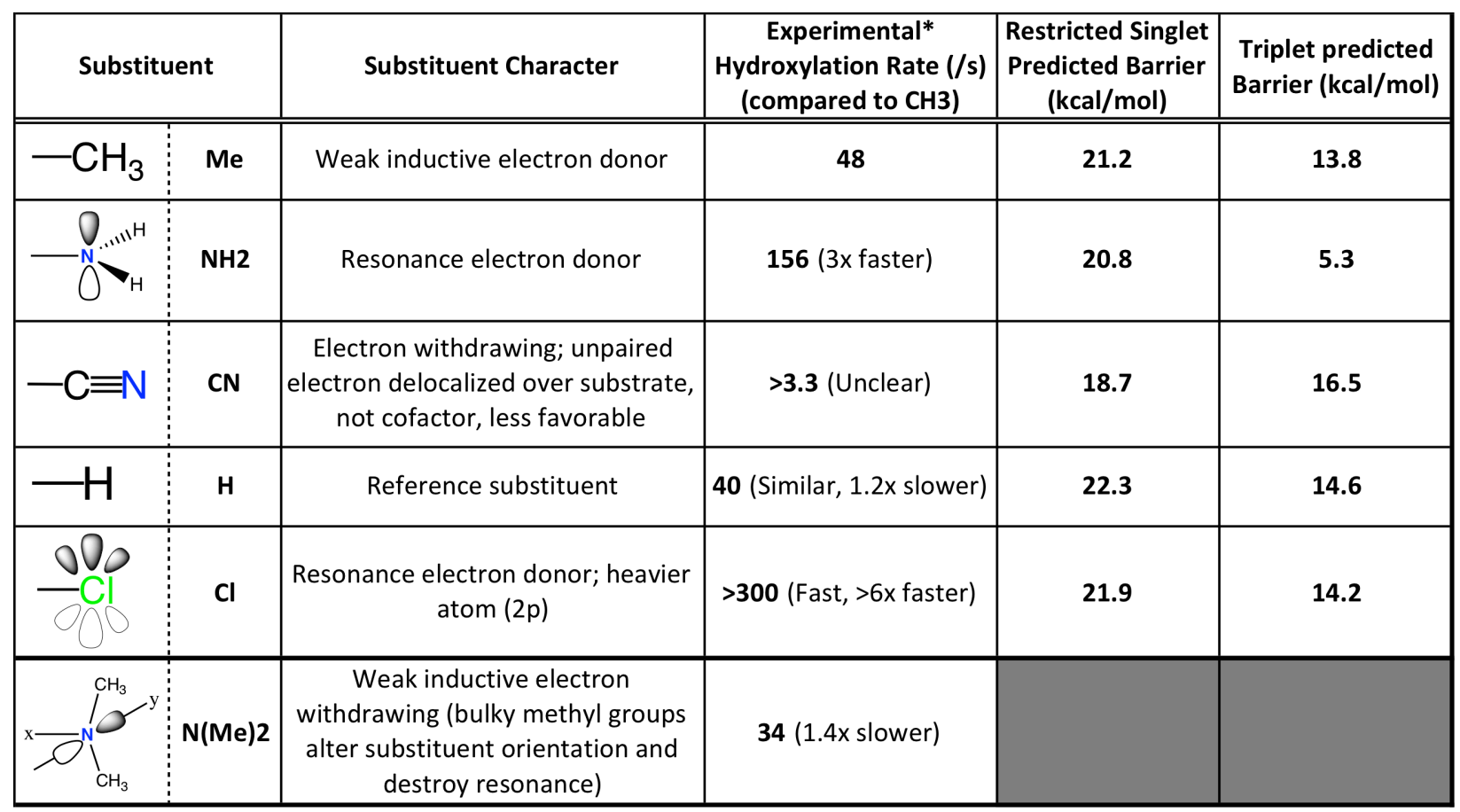

* Ortiz-Maldonado, M.; Ballou, D. P.; Massey, V. Use of Free Energy Relationships to Probe the Individual Steps of Hydroxylation of P-Hydroxybenzoate Hydroxylase: Studies with a Series of 8Substituted Flavins. Biochemistry 1999, 38, 8124-8137. 


\section{REFERENCES}

1. Brooks, B. R.; Brooks, C. L., III; Mackerell, A. D. Jr.; Nilsson, L.; Petrella, R. J.; Roux, B.; Won, Y.; Archontis, G.; Bartels, C.; Boresch, S., et al. Charmm: The Biomolecular Simulation Program. J. Comput. Chem. 2009, 30, 1545-1614.

2. Rodríguez Benítez, A.; Tweedy, S. E.; Baker Dockrey, S. A.; Lukowski, A. L.; Wymore, T.; Khare, D.; Brooks, C. L., III; Palfey, B. A.; Smith, J. L.; Narayan, A. R. H. Structural Basis for Selectivity in Flavin-Dependent Monooxygenase-Catalyzed Oxidative Dearomatization. ACS Catal. 2019, 3633-3640.

3. Lee, J.; Cheng, X.; Swails, J. M.; Yeom, M. S.; Eastman, P. K.; Lemkul, J. A.; Wei, S.; Buckner, J.; Jeong, J. C.; Qi, Y., et al. Charmm-Gui Input Generator for Namd, Gromacs, Amber, Openmm, and Charmm/Openmm Simulations Using the Charmm36 Additive Force Field. $J$. Chem. Theory Comput. 2016, 12, 405-413.

4. Gagnon, J. K.; Law, S. M.; Brooks, C. L, III. Flexible Cdocker: Development and Application of a Pseudo-Explicit Structure-Based Docking Method within Charmm. J. Comput. Chem. 2016, 37, 753-762.

5. Vanommeslaeghe, K.; Hatcher, E.; Acharya, C.; Kundu, S.; Zhong, S.; Shim, J.; Darian, E.; Guvench, O.; Lopes, P.; Vorobyov, I., et al. Charmm General Force Field: A Force Field for Drug-Like Molecules Compatible with the Charmm All-Atom Additive Biological Force Fields. J. Comput. Chem. 2009, 31, 671-690.

6. Vanommeslaeghe, K.; Mackerell, A. D. Charmm Additive and Polarizable Force Fields for Biophysics and Computer-Aided Drug Design. Biochim. Biophys. Acta 2015, 1850, 861-871.

7. Im, W.; Lee, M. S.; Brooks, C. L., III. Generalized Born Model with a Simple Smoothing Function. J. Comput. Chem. 2003, 24, 1691-1702.

8. Gaus, M.; Cui, Q.; Elstner, M. Dftb3: Extension of the Self-Consistent-Charge DensityFunctional Tight-Binding Method (Scc-Dftb). J. Chem. Theory Comput. 2012, 7, 931-948.

9. Kumar, S.; Bouzida, D.; Swendsen, R. H.; Kollman, P. A.; Rosenberg, J. M. The Weighted Histogram Analysis Method for Free-Energy Calculations on Biomolecules .1. The Method. $J$. Comput. Chem. 1992, 13, 1011-1021.

10. Perdew, J. P.; Burke, K.; Ernzerhof, M. Generalized Gradient Approximation Made Simple. Phys. Rev. Lett. 1996, 77, 3865-3868.

11. Becke, A. D. Density-Functional Thermochemistry .3. The Role of Exact Exchange. $J$. Chem. Phys. 1993, 98, 5648-5652.

12. Lee, C.; Yang, W.; Parr, R. G. Development of the Colle-Salvetti Correlation-Energy Formula into a Functional of the Electron Density. Phys. Rev. B: Condens. Matter 1988, 37, 785789.

13. Becke, A. D. Density-Functional Exchange-Energy Approximation with Correct Asymptotic Behavior. Phys. Rev. A: Gen. Phys. 1988, 38, 3098-3100.

14. Zimmerman, P. M.; Bell, F.; Goldey, M.; Bell, A. T.; Head-Gordon, M. Restricted Active Space Spin-Flip Configuration Interaction: Theory and Examples for Multiple Spin Flips with Odd Numbers of Electrons. J. Chem. Phys. 2012, 137, 164110.

15. Bell, F.; Zimmerman, P. M.; Casanova, D.; Goldey, M.; Head-Gordon, M. Restricted Active Space Spin-Flip (Ras-Sf) with Arbitrary Number of Spin-Flips. Phys. Chem. Chem. Phys. 2013, 15, 358-366. 
16. Chien, A. D.; Zimmerman, P. M. Recovering Dynamic Correlation in Spin Flip Configuration Interaction through a Difference Dedicated Approach. J. Chem. Phys. 2017, 146, 014103.

17. Altschul, S. F.; Madden, T. L.; Schaffer, A. A.; Zhang, J.; Zhang, Z.; Miller, W.; Lipman, D. J. Gapped Blast and Psi-Blast: A New Generation of Protein Database Search Programs. Nucleic Acids Res. 1997, 25, 3389-3402.

18. Huang, Y.; Niu, B.; Gao, Y.; Fu, L.; Li, W. Cd-Hit Suite: A Web Server for Clustering and Comparing Biological Sequences. Bioinformatics 2010, 26, 680-682.

19. Edgar, R. C. Muscle: Multiple Sequence Alignment with High Accuracy and High Throughput. Nucleic Acids Res. 2004, 32, 1792-1797.

20. Bailey, T. L. Fitting a Mixture Model by Expectation Maximization to Discover Motifs in Biopolymers. Proc. 2nd Int. Conf. ISMB 1994, 1-7.

21. Mardirossian, N.; Head-Gordon, M. Exploring the Limit of Accuracy for Density Functionals Based on the Generalized Gradient Approximation: Local, Global Hybrid, and RangeSeparated Hybrid Functionals with and without Dispersion Corrections. J. Chem. Phys. 2014, 140, 18 A527.

22. Glendening, E. D.; Landis, C. R.; Weinhold, F. Nbo 6.0: Natural Bond Orbital Analysis Program. J. Comput. Chem. 2013, 34, 1429-1437.

23. Woodcock, H. L.; Hodoscek, M.; Gilbert, A. T. B.; Gill, P. M. W.; Schaefer, H. F.; Brooks, B. R. Interfacing Q-Chem and Charmm to Perform Qm/Mm Reaction Path Calculations. J. Comput. Chem. 2007, 28, 1485-1502.

24. Ortiz-Maldonado, M.; Ballou, D. P.; Massey, V. Use of Free Energy Relationships to Probe the Individual Steps of Hydroxylation of P-Hydroxybenzoate Hydroxylase: Studies with a Series of 8-Substituted Flavins. Biochemistry 1999, 38, 8124-8137.

25. Ridder, L.; Mulholland, A. J.; Rietjens, I. M. C. M.; Vervoort, J. Combined Quantum Mechanical and Molecular Mechanical Reaction Pathway Calculation for Aromatic Hydroxylation by P-Hydroxybenzoate-3-Hydroxylase. J. Mol. Graphics Modell. 1999, 17, 163-175.

26. Ridder, L.; Harvey, J. N.; Rietjens, I. M. C. M.; Vervoort, J.; Mulholland, A. J. Ab Initio Qm/Mm Modeling of the Hydroxylation Step in P-Hydroxybenzoate Hydroxylase. J. Phys. Chem. B 2003, 107, 2118-2126.

27. Claeyssens, F.; Harvey, J. N.; Manby, F. R.; Mata, R. A.; Mulholland, A. J.; Ranaghan, K. E.; Schütz, M.; Thiel, S.; Thiel, W.; Werner, H.-J. High-Accuracy Computation of Reaction Barriers in Enzymes. Angew. Chem., Int. Ed. Engl. 2006, 45, 6856-6859.

28. Senn, H. M.; Thiel, S.; Thiel, W. Enzymatic Hydroxylation in P-Hydroxybenzoate Hydroxylase: A Case Study for Qm/Mm Molecular Dynamics. J. Chem. Theory Comput. 2005, $1,494-505$.

29. Bistoni, G.; Polyak, I.; Sparta, M.; Thiel, W.; Neese, F. Toward Accurate Qm/Mm Reaction Barriers with Large Qm Regions Using Domain Based Pair Natural Orbital Coupled Cluster Theory. J. Chem. Theory Comput. 2018, 14, 3524-3531.

30. Penfold, T. J.; Gindensperger, E.; Daniel, C.; Marian C. M., Spin-Vibronic Mechanism for Intersystem Crossing. Chem. Rev. 2018, 118, 6975-7025. 